\title{
Procesos de innovación en la industria manufacturera colombiana *
}

\author{
Innovation Processes in the Colombian Manufacturing Industry \\ Jorge Enrique Maldonado-Pinto \\ Doctor en Educación y Ciencias Administrativas, Corporación Nacional Unificada \\ de Educación Superior, \\ Cúcuta-Colombia, jorge_maldonado@cun.edu.co
}

Luisa Fernanda Portilla-Barco

Especialista en Alta gerencia, Corporación Nacional Unificada de Educación Superior, Cúcuta-Colombia, luisa.portilla23@gmail.com

\begin{abstract}
Cómo citar / How to cite
Maldonado-Pinto, J. E., \& Portilla-Barco, L. F. (2020). Procesos de innovación en la industria manufacturera colombiana. Revista CEA, 6(11), 145-163. https://doi.org/10.22430/24223182.1395
\end{abstract}

Recibido: 12 de junio de 2019

Aceptado: 15 de octubre de 2019

\section{Resumen}

El objetivo de este artículo es analizar los procesos de innovación en la industria manufacturera colombiana, para lo cual se utilizó como metodología la hermenéutica, ya que se interpretaron los resultados de las entrevistas y la revisión de algunos documentos. Entre los principales hallazgos, se estima que la industria manufacturera colombiana ha adquirido una cierta cultura de innovación; el talento humano de las organizaciones participa activamente en todos los procesos de innovación; en los trabajadores jóvenes se detecta una alta capacidad creativa y, finalmente, la innovación está presente en toda la gestión administrativa, en la actualización o mejora de los productos y se desarrolla de forma continua e incremental. Con respecto a la innovación tecnológica, los resultados arrojan que esta facilita el trabajo, si se cuenta con los equipos, la maquinaria y las herramientas adecuadas. La estrategia tecnológica es un proceso que involucra todo, especialmente, cuando el objetivo es la satisfacción total del cliente. También fue posible detectar que la estrategia tecnológica es una forma de pensar y constituye una política de innovación. Algunas compañías no protegen sus inventos con patentes, sin embargo, los más organizados sí lo hacen. En conclusión, la industria manufacturera colombiana innova en productos, procesos y servicios, y también apoya el desarrollo del sector con estudios de mercado y tendencias.

Palabras clave: procesos de innovación, industria manufacturera, competitividad, hermenéutica.

Clasificación JEL: O32, J24, L67.

\footnotetext{
*Este artículo se deriva del proyecto titulado «Procesos de Innovación en la Industria Manufacturera Colombiana» avalado como proyecto para la Corporación Unificada Nacional de Educación Superior, Universidad CUN y ha sido financiado con recursos propios.
} 


\begin{abstract}
This paper aims to analyze innovation processes in the Colombian manufacturing industry by adopting a hermeneutic methodology and analyzing the results of interviews and a literature review. The Colombian manufacturing industry has acquired a certain innovation culture where the human talent of organizations actively participates in all the innovation processes, young workers have a high creative capacity, innovation is embedded in administrative management (updating or improving products), and innovations are developed in a continuous and incremental way. These results show that technological innovation facilitates work if the appropriate equipment, machinery and tools are available. Furthermore, a technological strategy involves the entire organization, especially when the goal is total customer satisfaction. We also found that technological strategies are thinking styles that constitute a firm's innovation policy. Some companies do not protect their inventions with patents, but the most organized ones do. In conclusion, the Colombian manufacturing industry innovates products, processes, and services and supports the development of the sector with market studies.
\end{abstract}

Keywords: innovation processes, manufacturing industry, competitiveness, hermeneutics.

JEL classification: 032, J24, L67.

\title{
1. INTRODUCCIÓN
}

Mediante este trabajo se busca analizar los procesos de innovación que surgen en la industria manufacturera colombiana. Griffith et al., (2004) evidencian la importancia de las actividades I+D como factor determinante del crecimiento de la productividad en un grupo de industrias de doce países de la OCDE. La innovación se considera uno de los factores más relevantes de la productividad en las industrias y el que genera mayores utilidades en los negocios. Gracias a esta, las empresas crecen y se desarrollan, pues pueden ofrecer productos y servicios de calidad nuevos que satisfagan tanto las necesidades, deseos, gustos y preferencias generales de los consumidores como sus expectativas individuales.

En razón a esto, se exponen en el marco referencial tres (3) fundamentos teóricos que dan cuenta de la forma en que han evolucionado los procesos de innovación en el mundo. Con este fin, se toma como base a Martínez Villaverde (2010), que relaciona la innovación con la cultura y el cambio en el entorno como estrategia adoptada para el cambio y como proceso estratégico, de oportunidades, de generación de ideas y de gestión de proyectos, que requiere la protección de una patente.

El enfoque paradigmático es el cualitativo o interpretativo; el tipo de estudio es descriptivo y el método es inductivo, pues va de casos particulares (empresas entrevistadas) a la generalización. Así mismo, esta investigación expone los resultados de una revisión documental de los procesos de innovación en Colombia y aquellos derivados de las respuestas de las entrevistas. Por último, las conclusiones formuladas abarcan una serie de datos concretos y deducciones que ofrecen respuestas a los objetivos planteados desde el inicio de la investigación.

\section{MARCO TEÓRICO}

A continuación, se presenta una revisión bibliográfica para la comprensión de la temática, que inicia con la contextualización del problema, los antecedentes o el estado del arte y los referentes teóricos, para poder identificar los procesos de innovación en la industria manufacturera colombiana. 


\section{Contextualización de la problemática}

A partir del 7 de agosto de 2018, iNNpulsa (2019) —Unidad de Gestión de Crecimiento Empresarial del Gobierno de Colombia- asumió el compromiso de incentivar el emprendimiento, la innovación y la creatividad, porque, según su presidente en Colombia, Ignacio Gaitán, están convencidos de que el talento emprendedor e innovador de los colombianos puede transformar el país y lo único que necesitan es un ecosistema articulado que les brinde las opciones para crecer. De acuerdo con la revista Dinero (2018), Colombia invierte poco en innovación (0,7\% del PIB); del total de la inversión, apenas un $35 \%$ proviene del sector privado y menos del $22 \%$ de las empresas desarrollan procesos de innovación; en consecuencia, el país está atrasado en este tema y ocupa el puesto 65 entre 127 países, según el ranking Global Innovation Index (GII).

Según Morales et al., (2012), la investigación es un factor potencial de competitividad que ha impulsado a los gobiernos a implementar políticas macroeconómicas y sociales que repercutan en las capacidades estratégicas de las organizaciones. Sin embargo, dadas las trayectorias tecnológicas y económicas de los diferentes países, las brechas en la aplicación y apropiación de la innovación asociadas a las condiciones internas de las organizaciones y al contexto en que se desenvuelven son evidentes.

A lo largo de la historia, el hombre siempre ha buscado nuevas formas de hacer las cosas y de implementar ingeniosas herramientas que le permitan mejorar las condiciones de vida de la familia. Colombia, como país exportador de materias primas, recibe cada vez menos ingresos relativos al desaprovechar el impacto de la tecnología sobre el valor agregado de la transformación en productos terminados. Según la Asociación Nacional de Industriales (ANDI) (2019), en los últimos 18 años el crecimiento promedio de la productividad ha sido de apenas $0,5 \%$, lo que dificulta grandes avances en el desarrollo económico y social.

En esta dirección, es importante consolidar la concepción de cadena, fortalecer la estrategia de encadenamientos, avanzar en los temas de calidad, desarrollar el talento humano, invertir en ciencia, tecnología e innovación y tener siempre como referente los parámetros internacionales y el desarrollo de nuevos negocios. Busom (1993) afirma que la evidencia empírica de carácter microeconómico, apoya claramente la hipótesis de que la innovación tiene un efecto positivo sobre el crecimiento. Nawas Khan et al., (2019) encontraron una influencia positiva de la innovación sobre el desempeño organizacional. Autores como Pinto et al., (2019) afirman que la innovación juega un papel fundamental en el desarrollo económico y social. Para Sivam et al., (2019), la generación de nuevas ideas innovadoras ayuda a las organizaciones a alcanzar ventaja competitiva.

Finalmente, un problema importante del sector manufacturero colombiano es la falta de compromiso por parte de las empresas, respecto a los requerimientos de un mercado competitivo, es decir, la ausencia de políticas estructurales encaminadas a potenciar la industria para la competitividad internacional (López Riveros, 2013). Por este motivo es fundamental que los ejecutivos de las empresas sean conscientes de la relevancia de los procesos de innovación en las empresas (BID, 2016), pues sin esta no podrían ser competitivas en este mundo globalizado y globalizante (Bakator, et al., 2019). De acuerdo con los miembros de la ANDI, los principales agravantes en este punto - a saber, los factores que en mayor medida obstaculizan la dinámica del sector - son: el decrecimiento de la producción económica y el aumento del contrabando, debido al ingreso de productos ilegales al país como resultado de las aperturas comerciales; y la poca inversión extranjera, a causa de los problemas de seguridad y conflicto que ha tenido que afrontar el país por varios años (Chasqui Uribe y Soto Valderrama, 2016). 
Durante el año 2015, algunos problemas específicos que se presentaron fueron: el tipo de cambio seguido de los costos y suministros de las materias primas, los problemas de infraestructura y costos logísticos y el escaso compromiso por parte de las empresas respecto a los requerimientos de un mercado competitivo, es decir, la ausencia de políticas estructurales encaminadas a potenciar la industria para la competitividad internacional (López Riveros, 2013).

En razón a esto, es importante resaltar que los gerentes deben ser conscientes de la magnitud de los procesos de innovación en las empresas, frente a lo cual deben generar ambientes propicios que estimulen la creación de ideas y proyectos y el desarrollo de inventos o mejoras. Estos no deben quedar planteados solamente, sino que la empresa y, especialmente, los gerentes deben hacer lo posible para que lleguen a buen término.

Otro asunto que se destaca es la falta de apoyo del sector público, cuyo incentivo para las empresas innovadoras es difícil de alcanzar por las pequeñas organizaciones, ya que presentan mayores dificultades en este campo, razón por la cual las políticas de fortalecimiento deberían enfocarse principalmente en estas. Asimismo, la escasez de capital humano calificado dificulta la implementación de la innovación en las empresas, así como la diversidad en el tamaño y la falta de coordinación entre estas.

Con base en los argumentos expuestos, se puede colegir que la innovación en la industria manufacturera colombiana es aún incipiente, debido a varias razones, entre las cuales se encuentra la falta de una cultura de innovación generalizada, pues la mayoría de las empresas se dedican a explotar materias primas y no a transformarlas en productos terminados: algunas solo se dedican a copiar diseños y modelos de productos fabricados en el exterior.

Otra de las razones fundamentales es que muchas empresas no tienen un departamento de Innovación y Desarrollo (lyD), motivo por el cual las industrias manufactureras colombianas presentan un nivel bajo de innovación. Igualmente, existe un mercado globalizado y globalizante, en el que las telecomunicaciones facilitan el mercado internacional de productos y servicios, por lo que es muy difícil que la industria nacional pueda competir con la trasnacional.

De acuerdo con Díaz Rodríguez (2006), el objetivo de fortalecer y aumentar la viabilidad de la industria nacional en los diferentes sectores exige la alineación de capacidades, voluntades, políticas y recursos tanto de quienes constituyen y generan el entorno de la oferta como de la comunidad usuaria y, por lo tanto, principal promotora. De este modo, para el autor, se requieren acciones correctivas y preventivas que favorezcan el ambiente de desarrollo de las industrias.

\section{Estado del arte}

La innovación siempre ha jugado un papel importante en el éxito de las empresas industriales más competitivas, pero en los últimos años esto ha cambiado de manera radical, pues ya no es una opción, sino un imperativo en los negocios. Los mejores innovan de manera regular y la innovación del pasado no será suficiente en el futuro, en gran parte, debido a que la industria está experimentando una transformación fundamental a largo plazo. En 2025, una nueva clase consumidora habrá surgido y la mayor parte del consumo mundial se desarrollará en los países en vías de desarrollo, lo que se traduce en nuevas oportunidades (Ursúa Lasheras, 2019).

Estos cambios en la demanda coincidirán con la aplicación de grandes innovaciones en los materiales y procesos como los nanomateriales - en la integración de las TIC-, la impresión 3D y la robótica avanzada. Para adaptarse a estos cambios y seguir siendo competitivas, las empresas industriales 
necesitarán redefinir lo que significa la innovación para el negocio, para lo cual deben tener en cuenta que el margen de error se ha reducido.

En los últimos años, conforme se han ido reduciendo los márgenes de las empresas, se ha reducido la posibilidad de errar en las innovaciones que no cumplan con una determinada demanda de los clientes. Es un problema que se retroalimenta: debido a la reducción de márgenes, los fabricantes son menos propensos a asumir riesgos en la innovación; por otro lado, la innovación es un factor determinante para la diferenciación en muchas industrias en las que los compradores exigen productos y servicios innovadores. Para superar este reto, habrá que hacer la innovación más innovadora y fortalecer el enfoque innovador de las empresas, bajo la premisa de que la innovación debe ser omnipresente (Robayo Acuña, 2016).

La innovación no debe limitarse simplemente a la actividad de investigación y desarrollo. La empresa debe crear una cultura de innovación de toda la organización, para que esta sea realmente rentable. Según Morales y León (2014), un ejemplo relevante es el de la empresa Natura, líder en la fabricación y comercialización de cosméticos naturales de Latinoamérica. La innovación ha permitido que las ventas de esta gran empresa brasileña crezcan durante los últimos cinco años a una tasa anual del $73 \%$, cifra con la que muchas empresas de la región (y el mundo) apenas se atreven a soñar.

Estos resultados son clara evidencia de la gente y los procesos que Natura ha puesto en marcha para garantizar que las innovaciones no sean productos del azar, sino de un enfoque sistémico y disciplinado que involucra mentes creativas y talentosas y procesos gerenciales para su gestión.

Para crear una cultura de la innovación, se deben crear entornos de trabajo colaborativo y ofrecer a los empleados la oportunidad de liderar o participar en iniciativas de innovación importantes para que se desarrolle dicha cultura. Las empresas más innovadoras forman equipos interdisciplinares de alto rendimiento que trabajan en base a proyectos; a su vez, las tecnologías de la información (IT) tendrán un papel clave en este sentido. Los negocios industriales requerirán el uso de una nueva generación de sistemas ERP que se apoyen en tecnologías como croad computing, sistemas móviles, redes sociales y big data analytics.

Estas plataformas de IT permitirán crear entornos de colaboración y toma de decisiones en tiempo real que serán fundamentales para apoyar la implementación de estos procesos, en las organizaciones de todos los países. Para Morales et al., (2012), los países en vía de desarrollo evidencian una tendencia hacia la adquisición y adaptación de tecnologías externas como un factor importante y determinante en las capacidades de innovación, vistas desde una perspectiva netamente tecnológica. Esta ha sido la estrategia válida que ha hecho posible acercarse de manera práctica y más rápida a las experiencias de las organizaciones de países industrializados.

Oppenheimer (2014) plantea que en las empresas lo más importante es la gente; los trabajadores creativos e inclinados a la innovación producen más y con mayor eficiencia. Así mismo, afirma que es casi imposible que se creen empresas innovadoras en aquellos países cuyas legislaturas no favorecen la innovación y que un mal clima de negocios, las burocracias infernales y la corrupción son grandes trabas para este fin. Un claro ejemplo de esto es Venezuela, pues, según estudios del Banco Mundial, hacen falta 17 procesos legales para registrar una nueva empresa.

Según dataiFX (2017), en una encuesta aplicada por la ANDI, la gran mayoría de las empresas colombianas trabajan en la cultura de la innovación, pero no logran innovaciones. Es el momento de que se atrevan más, porque las lógicas globales no dan espera; es imperativo perder el miedo a equivocarse y entender el proceso como un aprendizaje. En razón a esto, el reto es equivocarse barato y corregir rápido. Puesto que no se puede ir a la velocidad del Estado, las empresas deben ver 
la financiación privada de la innovación como una herramienta para ser más competitivas y crecer; por esta razón, no deben depender únicamente de las convocatorias públicas.

La Ley de Spin Off (1838 de 2017) es una buena noticia para el país, ya que jalonará la creación de nuevas empresas de base tecnológica, con el objetivo de desarrollar más prototipos y lanzar al mercado productos mínimos viables que puedan ser cocreados con los consumidores, lo que ayuda a aminorar el riesgo de fracaso en el mercado. Para alcanzar estas metas, es importante contar con un sistema para gestionar la innovación, pues esta no puede ser un chispazo, sino que debe constituirse en un proceso recurrente, sistemático y deliberado. A continuación, en la tabla 1, se comparan algunas teorías de innovación.

Tabla 1. Comparación de teorías de innovación

Table 1. Comparison of innovation theories

\begin{tabular}{|c|c|c|}
\hline Autor & Nombre de la teoría & Variables \\
\hline \multirow{10}{*}{$\begin{array}{l}\text { Ponti y } \\
\text { Ferrás } \\
\text { (2008) }\end{array}$} & \multirow{10}{*}{ Pasión por innovar } & Innovación en la organización \\
\hline & & Innovación en la estructura \\
\hline & & Innovación en los procesos \\
\hline & & Espíritu imaginativo de las personas y equipos \\
\hline & & $\begin{array}{l}\text { Actitud creativa, renovada y constante de la } \\
\text { gente }\end{array}$ \\
\hline & & Perfil de las personas creativas \\
\hline & & Trabajar creativamente en equipo \\
\hline & & Macrotendencias del futuro \\
\hline & & $\begin{array}{l}\text { Gestión de la innovación en relación con el } \\
\text { entorno }\end{array}$ \\
\hline & & El empresario visionario \\
\hline \multirow{7}{*}{ Ponti (2010) } & \multirow{7}{*}{$\begin{array}{l}\text { Los siete movimientos de la } \\
\text { innovación }\end{array}$} & Rumbo \\
\hline & & Trabajo en equipo \\
\hline & & Cambio \\
\hline & & Creatividad \\
\hline & & Tendencia \\
\hline & & Proyecto \\
\hline & & Resultado \\
\hline \multirow{7}{*}{$\begin{array}{l}\text { Martínez } \\
\text { Villaverde } \\
\text { (2010) }\end{array}$} & \multirow{7}{*}{$\begin{array}{l}\text { Gestión del cambio y la } \\
\text { innovación en la empresa }\end{array}$} & $\begin{array}{l}\text { La innovación como relación con la cultura y el } \\
\text { cambio del entorno }\end{array}$ \\
\hline & & $\begin{array}{l}\text { La innovación como estrategia adaptada al } \\
\text { cambio }\end{array}$ \\
\hline & & $\begin{array}{l}\text { La innovación como proceso estratégico } \\
\text { planificado }\end{array}$ \\
\hline & & $\begin{array}{l}\text { La innovación como aprovechamiento de } \\
\text { oportunidades y generación de ideas }\end{array}$ \\
\hline & & $\begin{array}{l}\text { La innovación como parte del proceso de } \\
\text { gestión de proyectos }\end{array}$ \\
\hline & & La innovación como sistema de gestión \\
\hline & & $\begin{array}{l}\text { La innovación como resultado que requiere } \\
\text { protección de una patente }\end{array}$ \\
\hline
\end{tabular}

Fuente: elaboración propia a partir de (Ponti \& Ferrás, 2008; Ponti, 2010; Martínez Villaverde, 2010). 


\section{METODOLOGÍA}

El enfoque paradigmático es el cualitativo o interpretativo, a través del cual se busca analizar, interpretar y comprender los resultados de las entrevistas o de la investigación documental del objeto de estudio. Según Maldonado (2018), la investigación cualitativa se define como una categoría de diseños de investigación que extraen descripciones a partir de observaciones que adoptan la forma de entrevistas, narraciones, notas de campo, grabaciones, transcripciones de audio y video, registros escritos de todo tipo, fotografías, películas y artefactos.

Por la naturaleza de la investigación, el alcance de este estudio es de tipo descriptivo. Según Daulhe (citado por Fernández et al., 2003), los estudios descriptivos especifican las propiedades importantes de personas, grupos, comunidades o cualquier otro fenómeno que se someta a análisis.

La presente investigación es el resultado del trabajo de campo, que, en palabras de Cerda Gutiérrez (1993), es aquel que se realiza en contacto directo con la comunidad, grupo o personas que son motivo de estudio. Comúnmente el proceso de la recolección de datos a nivel social es el producto de un trabajo de campo. Para Arias (2012), la investigación de campo consiste en la recolección de datos directamente de la realidad donde ocurren los hechos, sin manipular o controlar variable e alguna.

A su vez, se utiliza el método inductivo, que consiste en recoger datos particulares para llegar a la generalización y que, según los planteamientos de Cegarra (2012), se basa en enunciados particulares - como las descripciones-, para plantear enunciados universales. De las entrevistas efectuadas a los informantes claves, se extraen las categorías, a fin de que sean interpretadas, analizadas y relacionadas entre sí, y deriven en las conclusiones claras de esta investigación.

El proceso de recolección de las entrevistas se desarrolló con base en preguntas semiestructuradas y respuestas abiertas, que tenían en cuenta las distancias; unas se hicieron de forma presencial y otras por medios tecnológicos en industrias manufactureras de Bogotá, Medellín, Cali, Barranquilla, Bucaramanga y Cúcuta. Se seleccionaron siete informantes clave de siete empresas seleccionadas, conformados por los directores o jefes del área de innovación de las empresas, gerentes o jefes comerciales, según el tamaño de la empresa. Para este fin, se tomaron las empresas señaladas como las más innovadoras del país, de acuerdo a dataiFX (2017).

\section{RESULTADOS}

A través del software Atlas-ti, se desarrollaron mapas conceptuales de cada una de las preguntas formuladas a los informantes claves pertenecientes a siete empresas, cuyos resultados de las categorías emergentes se exponen a continuación.

Frente a la pregunta, ¿̇la innovación hace parte de la cultura corporativa? (figura 1), los informantes claves ofrecieron las siguientes respuestas:

1) los trabajadores en conjunto tienen una alta capacidad creativa;

2) se hace innovación en productos, procesos y servicios, así como a través del nuevo método de comercialización que implica cambios significativos del diseño o de la presentación de los productos, en su posicionamiento o en su promoción;

3) se crean, además, nuevos mercados;

4) la innovación está presente en toda la gestión administrativa y responde a cualquier actualización o mejora en los productos existentes de forma continuada y 
5) la innovación no supone ni necesita de cambios en el comportamiento para el consumo, pues hace parte fundamental de la cultura corporativa.

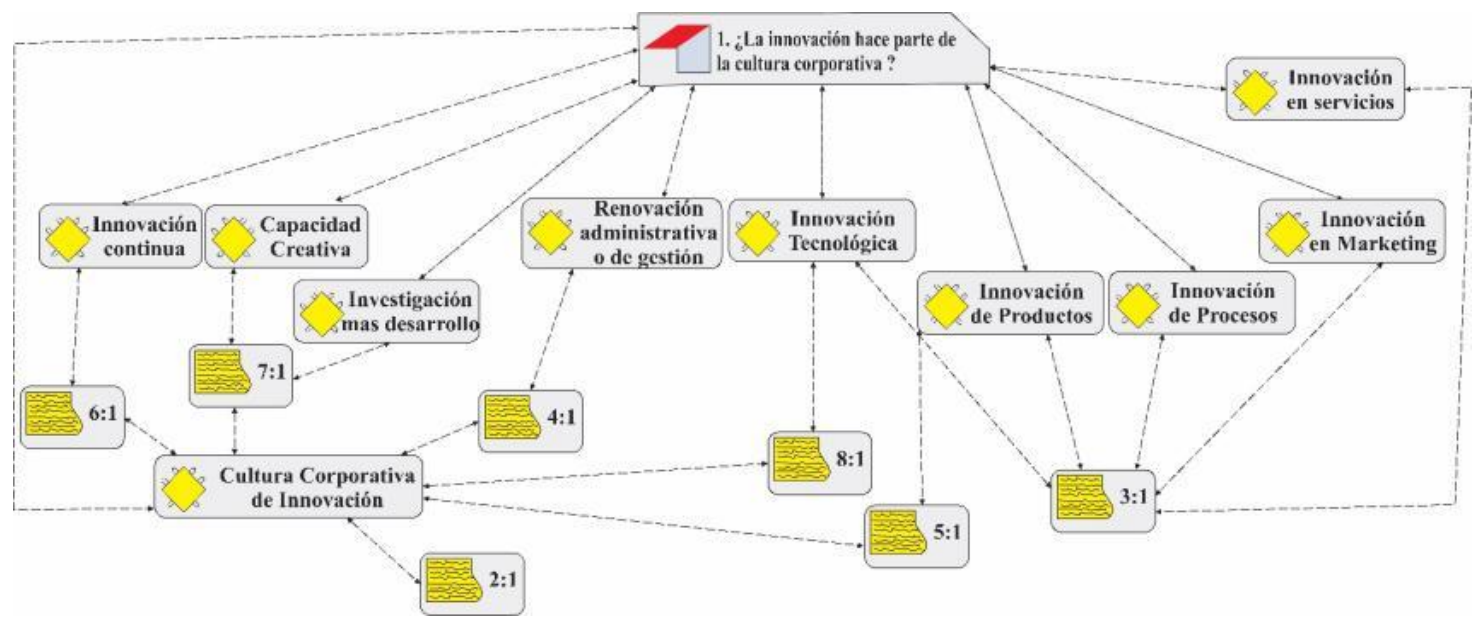

Figura 1. ¿La innovación hace parte de la cultura corporativa?

Figure 1. Is innovation part of the corporate culture?

Fuente: elaboración propia.

Ante la pregunta, ¿su organización considera la innovación como un proceso? (figura 2), los informantes clave de las empresas entrevistadas respondieron:

1) sí, es el proceso de formación para la compañía;

2) tenemos un proceso de inducción y damos una amplia capacitación e incentivamos a los trabajadores para que innoven en los puestos de trabajo;

3) a través de los equipos de trabajo, y orientados por el área de Investigación y Desarrollo, los trabajadores hacen innovación en los procesos de forma continuada: innovación incremental e innovación en nuevos mercados;

4) se hacen mejoras en la imagen, apariencia o costo y

5) se considera como innovación incremental cuando se crea un valor sobre un producto que ya existe, añadiéndole nuevas mejoras.

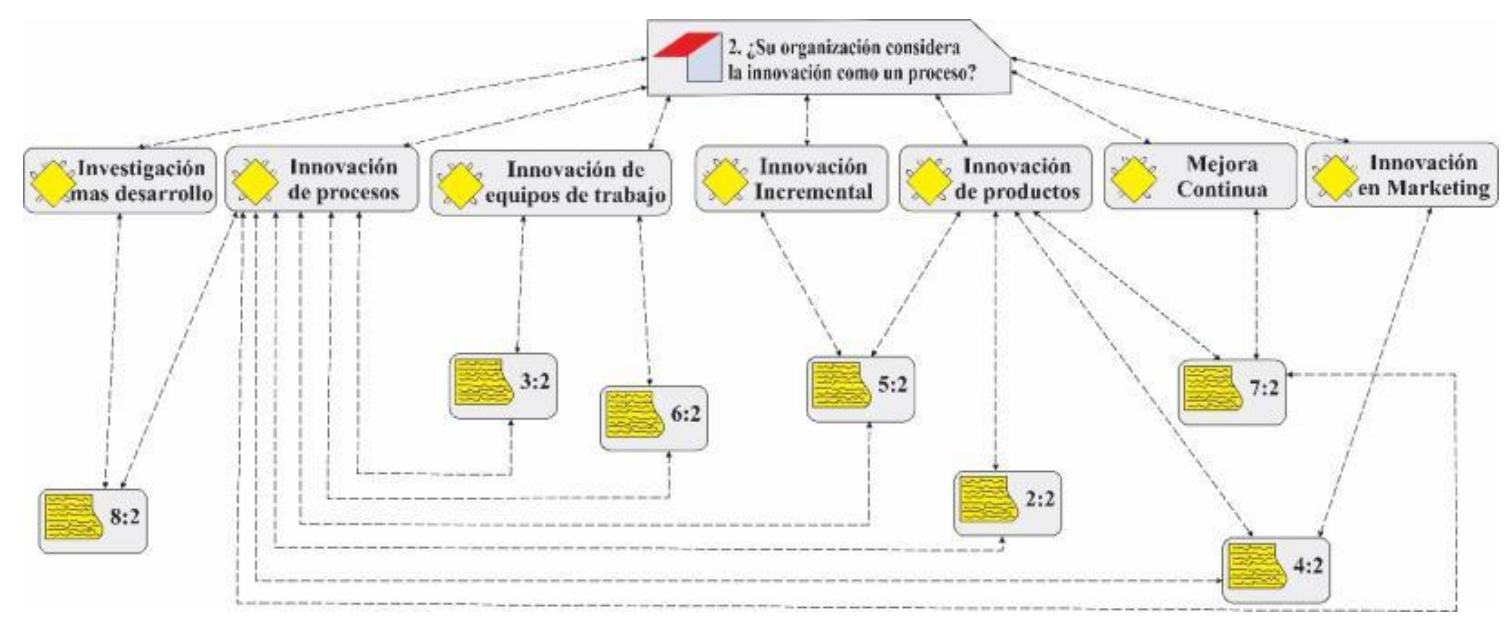

Figura 2. ¿Su organización considera la innovación como un proceso?

Figure 2. Is innovation considered a process in your organization?

Fuente: elaboración propia. 
Ante la pregunta, ¿considera usted que la estrategia tecnológica afecta la innovación corporativa? (figura 3), los representantes de las empresas entrevistadas contestaron:

1) la innovación tecnológica facilita el trabajo, lo hace más eficiente;

2) con la compra de tecnologías, se refuerza el área de modernización de la planta;

3) las herramientas tecnológicas facilitan el trabajo;

4) las herramientas tecnológicas facilitan el trabajo en relación con el cumplimiento de las normas ISO;

5) sí considera que la estrategia tecnológica afecta positivamente la innovación corporativa;

6) sí, la estrategia tecnológica hace parte de la innovación corporativa;

7) es un pilar de la innovación;

8) la estrategia tecnológica es una forma de pensar, es una política de innovación;

9) hace parte del avance tecnológico, es el proceso que involucra todo y cuyo objetivo principal es la satisfacción total del cliente y

10) todos los trabajadores poseen la capacidad para innovar y tratan de hacerlo al menor costo posible.

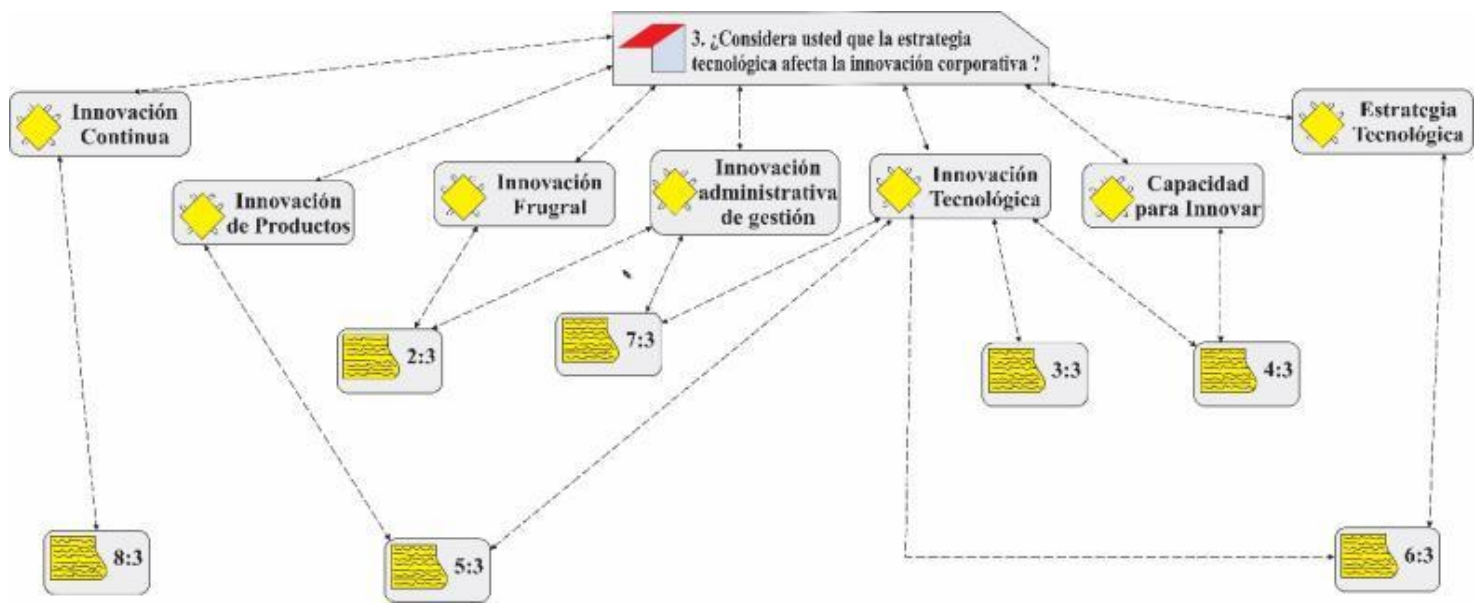

Figura 3. ¿Considera usted que la estrategia tecnológica afecta la innovación corporativa?

Figure 3. Do you think technological strategies affect the corporate culture?

Fuente: elaboración propia.

En cuanto al impacto de la creatividad en la cultura de la innovación (figura 4), las respuestas fueron:

1) se incentiva mucho la creatividad en los trabajadores;

2) su mayor fortaleza son los trabajadores jóvenes;

3) existe una cultura abierta a la creatividad;

4) no se castiga el error, se incentivan las nuevas formas de hacer las cosas;

5) los colaboradores trabajan en equipo, tienen capacidad creativa, hacen innovación en nuevos negocios y 6) la creatividad sí impacta la cultura de la innovación. 


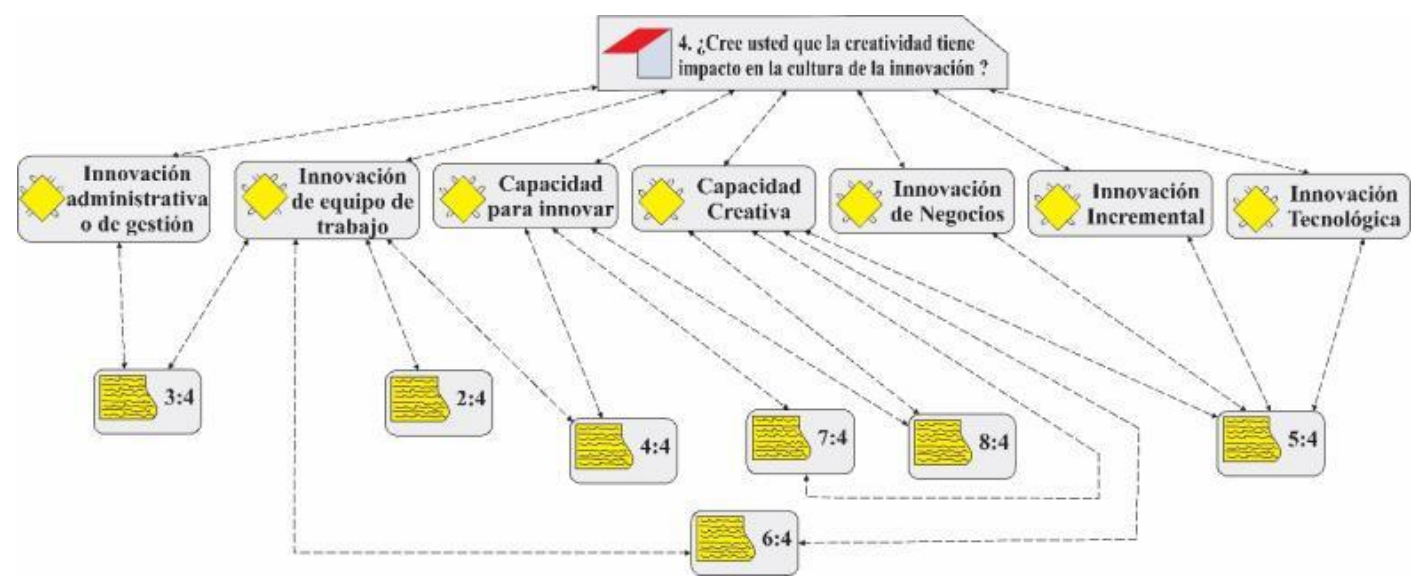

Figura 4. ¿Cree usted que la creatividad tiene impacto en la cultura de la innovación?

Figure 4. Do you think creativity has an impact on the innovation culture?

Fuente: elaboración propia.

En lo referente a la gestión de proyectos como una importante herramienta para la innovación (figura 5), los entrevistados contestaron:

1) es muy importante la gestión de proyectos, para la empresa, tiene una visión muy grande;

2) lo que se vende es la satisfacción del cliente, no los productos;

3) hay que escuchar al cliente, para poder brindarle productos y servicios de calidad que lo satisfagan;

4) de acuerdo a la necesidad del mercado, se fabrican los productos que el cliente necesita, por lo tanto, la gestión de proyectos sí es una importante herramienta para la innovación;

5) la cocreación con el cliente en proyectos individuales y personalizados se considera una herramienta esencial para la innovación de productos, procesos y servicios.

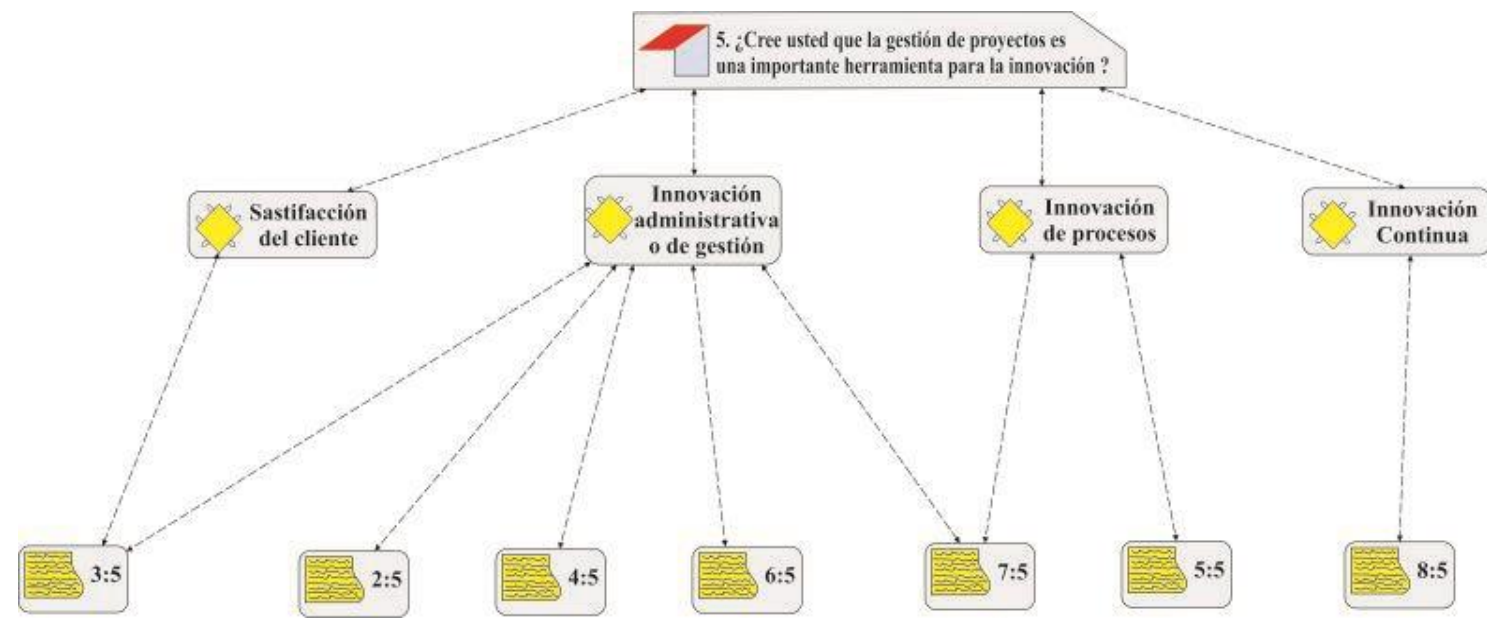

Figura 5. ¿Usted cree que la gestión de proyectos es una importante herramienta para la innovación? Figure 5. Do you think project management is an important tool for innovation? Fuente: elaboración propia.

Frente a la importancia de la adopción de un sistema de gestión de innovación para el crecimiento de la empresa (figura 6), las respuestas fueron:

1) sí, la adopción de un sistema de gestión de innovación es una herramienta muy importante para el crecimiento de la organización, pues revisa la calidad de los resultados que se están entregando; 2) sí, porque los sistemas de gestión de innovación nos permiten organizar y direccionar los recursos tanto humanos como económicos, a fin de incrementar la generación de ideas técnicas para obtener nuevos productos y procesos y mejorar los ya existentes; 
3) es la manera de asegurar que la innovación no ocurre por azar, sino que obedece al desarrollo de una capacidad sistemática y sostenible para innovar;

4) incluso hay sistemas de gestión de la calidad, basados en normas ISO, que pueden y deben incorporar la gestión de la innovación como un capítulo o como todo un sistema completo y

5) es muy importante, porque permite el seguimiento en la ejecución de las ideas y obras a implementar.

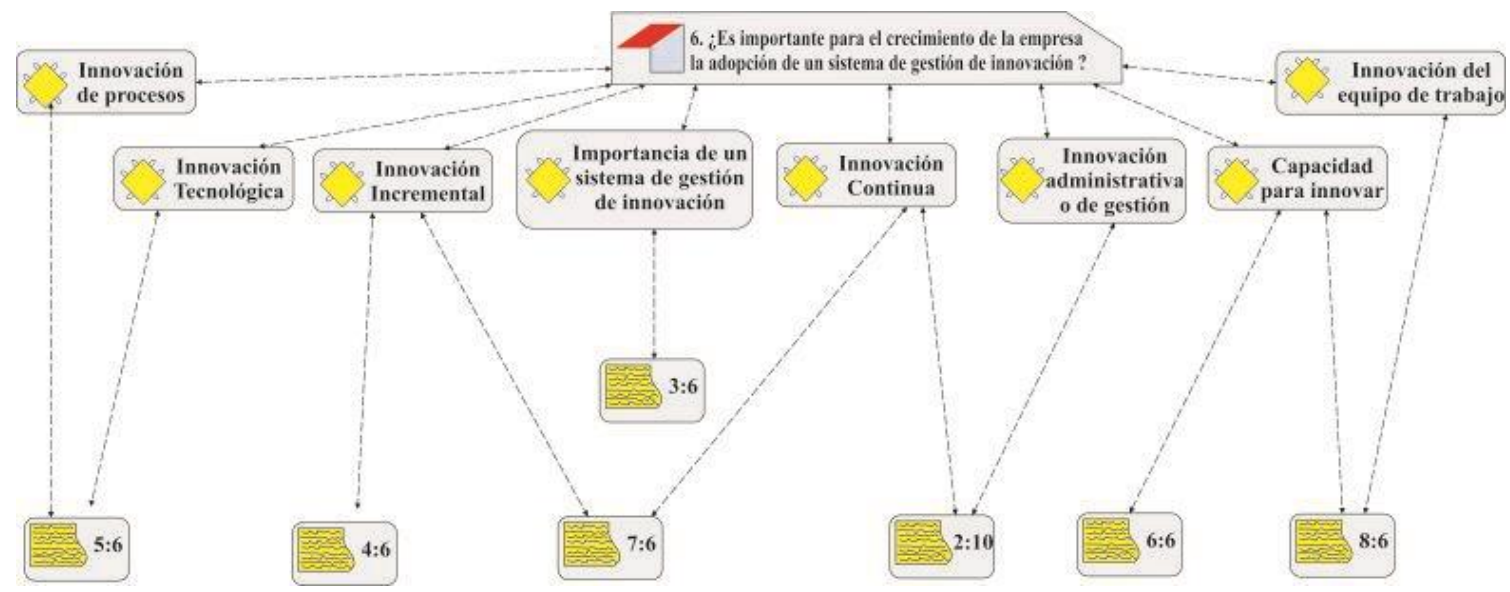

Figura 6. ¿Es importante para el crecimiento de la empresa la adopción de un sistema de gestión de innovación?

Figure 6 . Is the adoption of an innovation management system important for a company's growth? Fuente: elaboración propia.

Sobre la protección con patentes de los resultados de los procesos de innovación (figura 7), el personal que tienen que ver con los procesos de innovación de las industrias manufactureras entrevistadas contestó que algunas de las industrias manufactureras organizadas en Colombia protegen con patentes los resultados de los procesos de innovación; sin embargo, las empresas pequeñas y poco organizadas no lo hacen.

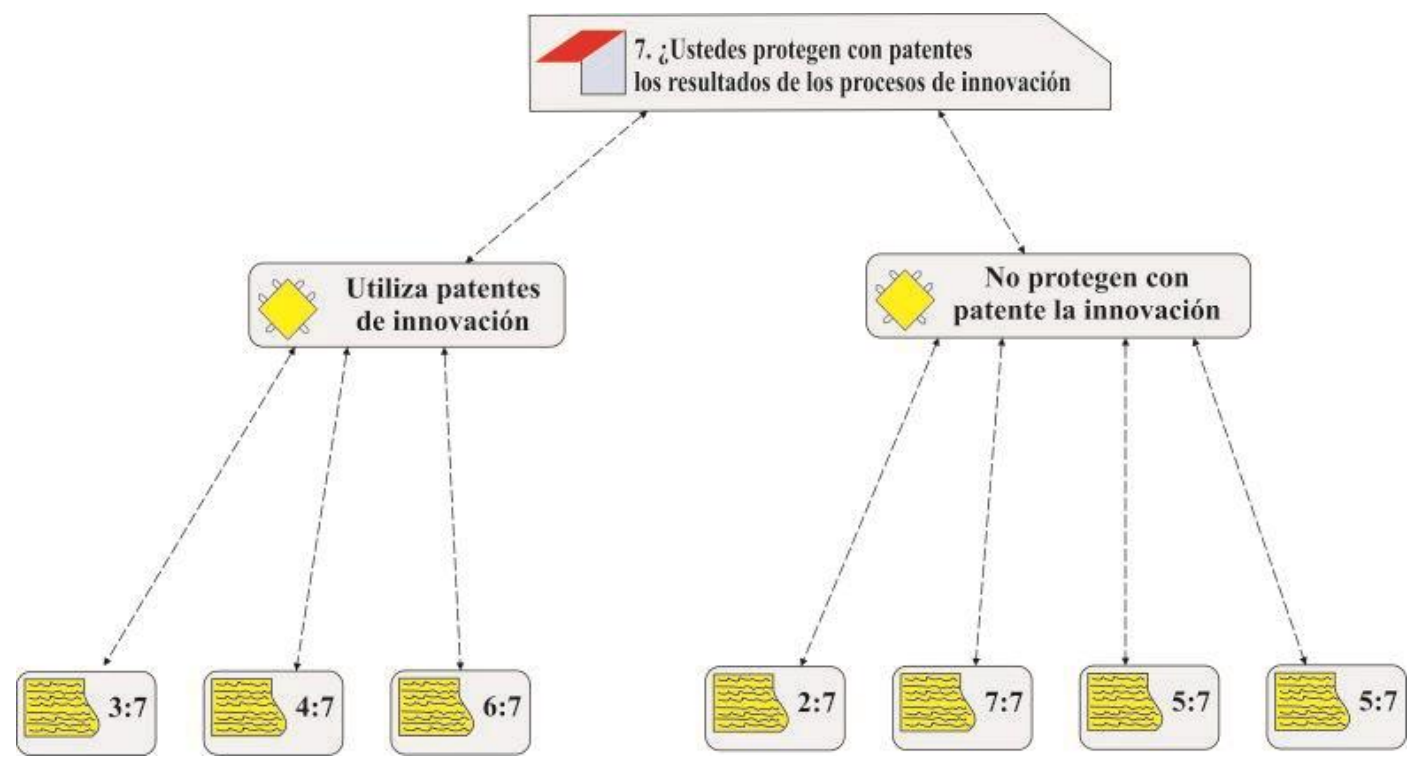

Figura 7. ¿Ustedes protegen con patentes los resultados de los procesos de innovación? Figure 7. Do you protect the results of innovation processes with patents? Fuente: elaboración propia. 
Ante la pregunta: ¿ustedes hacen innovación de sus productos, servicios y procesos? (figura 8), los informantes clave contestaron:

1) sí, somos fuertes en innovación tecnológica, los equipos que se utilizan son de tecnología de punta $y$, a través de ellos, se ha generado tecnología disruptiva que ha permitido un menor costo en los procesos y el mejoramiento en la realización de tareas para tener una mejor oferta para los clientes; 2) somos fuertes en innovación de productos -en servicios no tanto- y en procesos se está trabajando;

3) se crea el área de cultura de la innovación para mejorar los procesos y

4) la innovación que se hace de productos, servicios y procesos está orientada a la satisfacción plena del cliente.

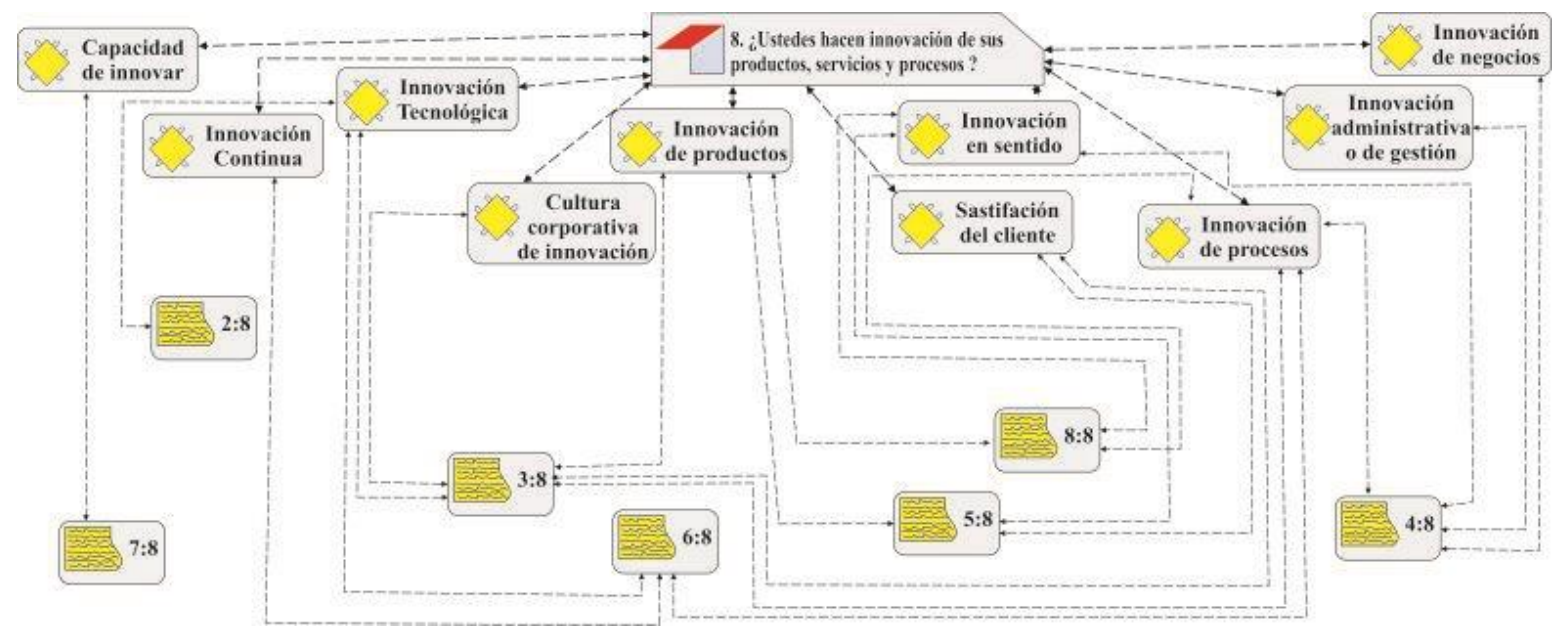

Figura 8. ¿Ustedes hacen innovación de sus productos, servicios y procesos?

Figure 8. Do you innovate your products, services, and processes?

Fuente: elaboración propia.

Respecto a la forma en que innovan en productos, servicios y procesos (figura 9), los informantes opinaron:

1) sí hacen innovación en productos, servicios y procesos, con base en los estudios de mercado y tendencias de diseño y decoración;

2) procuran la atención y satisfacción de los clientes;

3) usan talento humano de los trabajadores, herramientas, procesos, habilitadores tecnológicos y know how;

4) algunas de las herramientas son: Design Thinking, Sit, Six Sigma, Lean startup, así como el desarrollo de nuevos productos y Agile;

5) efectúan exhibiciones de los productos nuevos en espacio real para impactar a los clientes;

6) para la mejora y el desarrollo de nuevos productos, cuentan con un departamento de diseño, el cual propone ideas, alternativas y soluciones encaminadas a resolver las necesidades de los clientes; 7) las ideas son analizadas, evaluadas y llevadas a la realidad, en el departamento de proyectos, para lo cual siguen en la estructura de las cinco fases de la gestión de proyectos;

8) para la implementación de mejoras en los procesos productivos se sigue la misma metodología de análisis y ejecución;

9) se hace innovación continua e incremental;

10) se buscan nuevas tecnologías para mejorar tanto los productos como los procesos;

11) se está mejorando los servicios de preventa y posventa, debido a los clientes de otros mercados como Asia y África y 
12) a través del área de innovación corporativa, se están consiguiendo los recursos necesarios para la transformación y modernización de los procesos en cuanto a servicios y todo lo que tiene que ver con la atención y satisfacción del cliente.

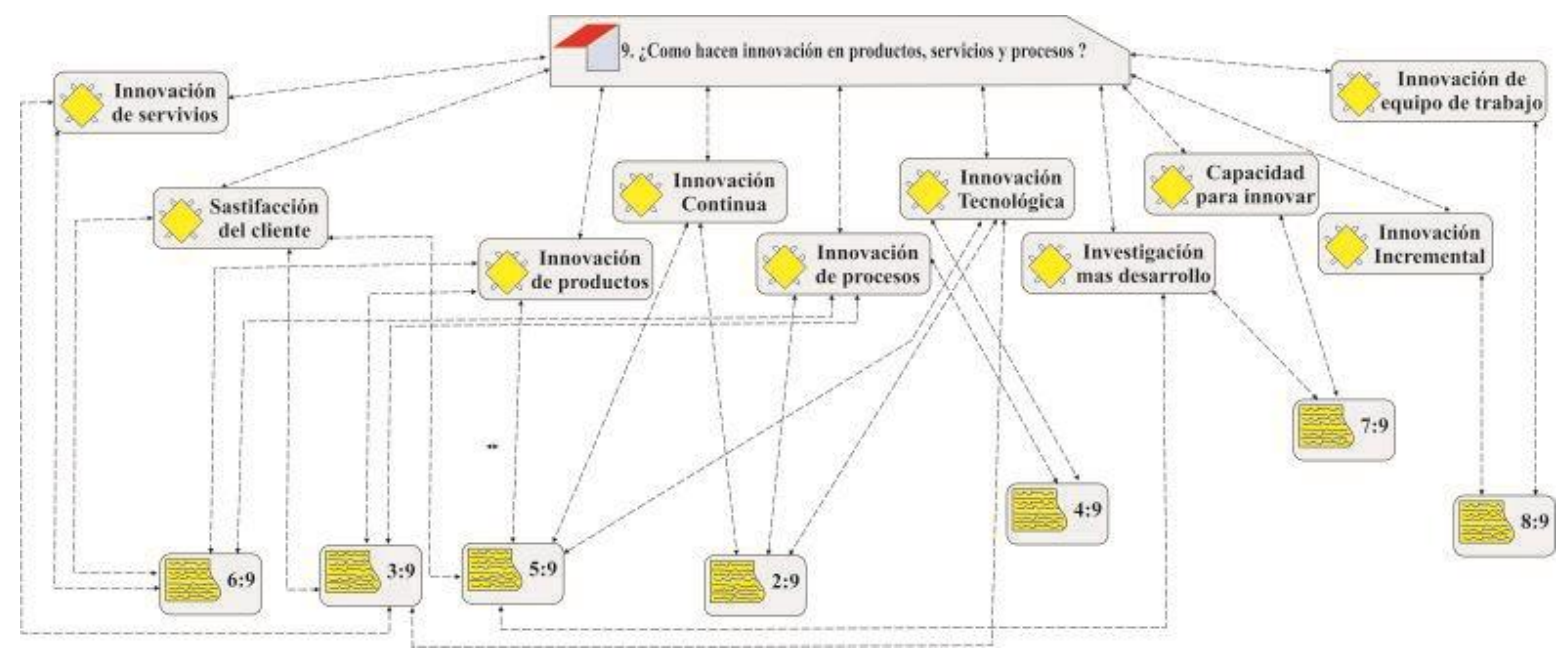

Figura 9. ¿Cómo hacen innovación en productos, servicios y procesos?

Figure 9. How do you do innovate your products, services and processes? Fuente: elaboración propia.

A continuación, en la tabla 2, considerada una de las más importantes de la investigación realizada, se muestran las categorías emergentes, resultado de la interpretación y análisis de los mapas conceptuales que el software Atlas-ti arrojó al aplicarlo a las respuestas de los representantes o directivos (informantes clave), de las empresas colombianas que se entrevistaron.

Tabla 2. Categorías emergentes

Table 2. Resulting categories

\# Categoría Explicación del concepto

1 Innovación tecnológica

2 Innovación en procesos

Innovación en
productos

$4 \quad$ Innovación en marketing

$5 \quad$ Innovación de negocio
La innovación tecnológica se refiere a la creación de un nuevo producto o servicio, siempre dirigido a los consumidores. En otras palabras, lo que se quiere decir es que existe innovación cuando las empresas presentan en el mercado nuevos elementos o, en su defecto, mejoran los ya existentes. Aunque esta innovación suele ir dirigida a la comercialización del producto o servicio, también puede relacionarse con los procesos de producción en la empresa.

Definición de nuevos procesos, o la mejora de los existentes, encaminada a incrementar el nivel de eficiencia de la empresa. Innovación en la gestión: mejoras en las actividades de gestión de la empresa (logística, administración, almacén, etc.)

Consiste en la introducción en el mercado de un bien o un servicio nuevo o sensiblemente mejorado con respecto a sus características básicas, especificaciones técnicas, software incorporado y otros componentes intangibles, finalidades deseadas o prestaciones.

La aplicación de un nuevo método de comercialización que implique cambios significativos del diseño o el envasado de un producto, su posicionamiento, su promoción o su tarificación. Nuevos mercados.

Es el proceso de crear, adaptar o traducir una idea, tecnología, herramienta, modelo o metodología en un bien o servicio que crea valor económico, por el cual los clientes pagarían. Creación de un nuevo negocio, estructuración de un modelo que incluye la propuesta de valor, sistemas de operación y análisis financiero. 


\begin{tabular}{cll} 
\# & Categoría & \multicolumn{1}{c}{ Explicación del concepto } \\
6 & $\begin{array}{l}\text { Innovación } \\
\text { administrativa o } \\
\text { de gestión }\end{array}$ & $\begin{array}{l}\text { También denominada innovación organizacional. Busca introducir en la empresa } \\
\text { nuevas herramientas administrativas que van desde la técnica de gestión hasta la } \\
\text { explicación de nuevos modelos gerenciales. }\end{array}$
\end{tabular}

Consiste en un tipo de innovación empresarial basada en aumentar el valor de un producto o servicio. Mejoras en su imagen, apariencia o costo. Se considera innovación incremental cuando se crea un valor sobre un producto que ya existe y se le añaden nuevas mejoras. El proceso de innovación comienza sobre una base conceptual. A partir de aquí, se desarrolla una serie de procesos creativos enfocados a conseguir unos fines determinados.

Innovación

8 continua o

adaptativa

$9 \quad$ Innovación en

servicios

Investigación y desarrollo

11 Innovación frugal

Innovación en equipos de trabajo

Capacidad creativa innovar

Cultura corporativa de innovación tecnológica cliente

Importancia de un sistema de gestión de innovación
Consiste en hacer pequeñas mejoras sistemáticas en productos y servicios. Responde a cualquier actualización o mejora en los productos existentes en forma continuada que, por sí misma, no suponga ni necesite de cambios en el comportamiento para su consumo.

Es el incremento o mejora de los servicios ya existentes.

Es el conjunto de actividades emprendidas de forma sistemática, a fin de aumentar el caudal de conocimientos científicos y técnicos, así como la utilización de los resultados de estos trabajos para conseguir nuevos dispositivos, productos, materiales o procesos.

La innovación frugal consiste en hacer más con menos. El nacimiento de pequeñas empresas ha favorecido que aquellos emprendedores que deseen mantener sus negocios a pesar de la competencia, deban idear estrategias de bajo coste que les permitan aprovechar sus recursos para innovar y desarrollar productos o servicios sin necesidad de involucrar un importante capital.

Se produce cuando el personal trabaja en equipo y cada uno aporta algo diferente a la solución de los problemas; su resultado es una sinergia enriquecedora.

La creatividad es la capacidad de generar nuevas ideas o conceptos, de nuevas asociaciones entre ideas y conceptos conocidos, que habitualmente producen buenos resultados para la organización.

La capacidad de innovación es una competencia distintiva que permite innovar y, en consecuencia, potencia la competitividad, especialmente en las pequeñas y medianas empresas (pyme). Dicha capacidad se ve condicionada por factores internos y externos.

Una empresa genuinamente innovadora es la que decide invertir tiempo y dinero en emprender nuevos proyectos y promover la creatividad y el aprendizaje continuo de sus trabajadores.

Es el proceso de adopción y ejecución de decisiones sobre las políticas, estrategias, planes y acciones relacionadas con la creación, difusión y uso de la tecnología.

Es un estado de felicidad por parte del cliente al conseguir la plena satisfacción de acuerdo a sus deseos, gustos y preferencias y expectativas por la adquisición de un producto o servicio.

Las empresas, al tener un sistema de gestión de innovación, llenan las expectativas de trabajo de todos los colaboradores, lo que deriva en una organización más competitiva en el mercado.

Las patentes de innovación son importantes para la seguridad legal de las propiedades de todas las creaciones o invenciones, ya sean en productos, procesos o servicios. 


\begin{tabular}{cll}
\hline$\#$ & Categoría & \multicolumn{1}{c}{ Explicación del concepto } \\
\hline 20 & $\begin{array}{l}\text { No protegen con } \\
\text { patentes de } \\
\text { innovación }\end{array}$ & $\begin{array}{l}\text { Al no proteger la creación de nuevos productos, servicios y procesos con patentes } \\
\text { debidamente registradas, ponen en riesgo legal la propiedad de todas sus } \\
\text { innovaciones. }\end{array}$ \\
\hline
\end{tabular}

Fuente: elaboración propia.

\section{DISCUSIÓN}

\section{Procesos de innovación en la industria manufacturera colombiana}

Se considera proceso a un conjunto de operaciones, a través de etapas sucesivas en relación con un hecho concreto o fenómeno. El proceso de innovación implica cambios significativos en productos y procesos (Gallardo-Vázquez et al., 2019). Para Egbu y Young (1998), «la innovación es la explotación exitosa de las ideas», por lo que se estima como el proceso de transformar ideas en valor para la empresa y los consumidores. Es por esto que se considera la innovación como

El proceso de transformar ideas en valor para la organización y los consumidores, el cual se inicia con la generación de ideas, pasando por un tamizaje de viabilidad, hasta la implementación de un nuevo, o significativamente mejorado: producto -bien o servicio-, proceso, esquema de mercadeo o estructura organizacional de la empresa (García González, 2012, p. 4).

La mayoría de los representantes de las industrias manufactureras colombianas entrevistados manifestaron que hacen innovación en la gestión administrativa, en el mejoramiento de productos actuales y nuevos, en algunos servicios y en nuevos negocios y mercados. Igualmente, expresaron que, a través del área de innovación corporativa, se están consiguiendo los recursos necesarios para la transformación y modernización de los procesos. En cuanto a los servicios, es una preocupación constante todo lo que tiene que ver con la atención y satisfacción del cliente, así como la entrega oportuna de los productos. En lo referente a los procesos industriales, se está mejorando la calidad y la efectividad en la producción.

Uno de los hallazgos más relevantes en esta investigación es que la mayoría de las industrias manufactureras colombianas no cuentan con patentes de innovación. Si bien esa carencia es común a la mayoría de las empresas colombianas estudiadas (Durán, Ibáñez, Salazar y Vargas, 2000; Malaver Rodríguez y Vargas Pérez, 2004), es importante revisar con más cuidado la relación entre el desarrollo organizacional, administrativo y de los procesos de innovación. Son muy pocas las industrias que cuentan con departamentos de I+D o con dependencias formales que hagan sus veces y con un contexto y grado de desarrollo administrativo que se preocupe por tener al día y cumplir con la normatividad existente en la gestión tanto administrativa como tecnológica. Cabe aclarar que, en algunas empresas con un nivel de organización mayor, sí cumplen con estos aspectos, como es el caso de Sumicol (perteneciente al Grupo Corona), Nalsani Toto, Industrias Haceb, Colcerámica, entre otras.

Todo lo anterior ha establecido condiciones muy favorables para la creación de nuevas invenciones y patentes destinadas a mejorar los procesos, los servicios y los procedimientos administrativos, y a desarrollar la innovación en nuevos negocios y marketing. En Colombia existen empresas en pleno proceso de crecimiento que pueden encontrarse desprotegidas, si sus innovaciones no están patentadas en el país o en otras naciones.

Para cualquier industria es prioritario que sus innovaciones cuenten con una patente que las respalde y proteja ante terceros, para evitar que hagan de esas invenciones un uso no autorizado. Además, el registro oportuno de las patentes puede generar a mediano plazo un significativo incremento de 
ingresos, si la invención tiene una alta utilidad o aplicabilidad para la competencia. Si las empresas desean cumplir con el objetivo de llegar a otros mercados, es fundamental entrar con sus patentes, especialmente para aquellas que deseen instalar sus operaciones en otros países.

Según los resultados del índice global de innovación del año 2018, la industria manufacturera colombiana sí ha adquirido una cierta cultura de la innovación. Esto es muy esperanzador, pues Colombia ocupa el puesto 63 a nivel mundial, por delante de países como Brasil, Panamá y Argentina. Uno de sus mayores logros es el reconocimiento, por primera vez en la historia, como un país innovation achiever, es decir, un país cuyo desempeño en innovación es superior con respecto a su nivel de desarrollo.

El valor agregado de esta investigación es el diagnóstico exploratorio de los procesos de innovación en las empresas participantes y la exposición de los logros y las expectativas del país en cuanto a este tema de tan alta relevancia. En razón a lo anterior, se plantean a continuación algunas recomendaciones:

- Capacitar constantemente al personal de la industria manufacturera colombiana en procesos de innovación y creatividad.

- Las empresas manufactureras colombianas deben renovar constantemente maquinarias y equipos y ponerse a la vanguardia en innovación tecnológica.

- Organizar y preparar equipos de trabajo eficientes. El poder de la mente colectiva es poderoso y, asimismo, la cocreación con los clientes en la innovación de productos y servicios.

- La opinión del cliente es supremamente importante.

Para Juan Carlos Garavito Escobar, gerente general de inNpulsa (2018), todo esto demuestra que seguimos avanzando en la apropiación de la innovación como una herramienta esencial para diversificar nuestra economía, gracias al esfuerzo conjunto de entidades del sector público y privado, que cada vez apuestan más por el desarrollo innovador en pro de la transformación del aparato productivo de Colombia.

\section{CONCLUSIONES}

En la industria manufacturera colombiana tanto empresarios como trabajadores han adquirido cierta cultura de la innovación. Sobre la innovación tecnológica, los informantes clave manifestaron que facilita el trabajo y lo hace más eficiente, razón por la cual, de cierta forma, afecta positivamente la innovación corporativa. Para las organizaciones, la estrategia tecnológica es una forma de pensar, una política de innovación, un proceso que involucra todo y cuyo objetivo principal es la satisfacción total del cliente. Según los entrevistados, la gestión de proyectos es muy importante para la empresa porque, gracias a esta, adquiere una visión muy grande; así, se configura como una importante herramienta para la innovación.

Por otra parte, es preocupante que algunas empresas manufactureras en Colombia no protejan con patentes sus invenciones; sin embargo, las empresas más organizadas sí lo hacen como parte de una cultura de innovación. De acuerdo a la OCDE, las patentes son un indicador válido para el tema de la innovación y sus datos, los cuales son fáciles de obtener, ya que están registrados en oficinas determinadas como la Superintendencia de Industria y Comercio de Bogotá. En este caso, lo difícil es interpretar dichos registros, razón por la cual la OCDE (2009) actualizó el Manual de Estadísticas y Patentes, con el objetivo de clarificar la utilización dada a estos indicadores y compararlos entre sí como elementos de medición. 
Finalmente, las empresas entrevistadas, a través de sus informantes clave, expresaron que sí hacen innovación en productos, servicios y procesos y que, además, lo hacen con base en investigaciones de mercados y en tendencias en diseño, moda, decoración y oportunidades de mercados. Principalmente, se innova a nivel adaptativo e incremental. La innovación adaptativa o transformadora consiste en esperar el cambio y cambiar las reglas del sector, con lo cual se modifica el contexto competitivo; utiliza, además, el talento humano de sus trabajadores, las herramientas, los equipos, los procesos, los habilitadores y el know how. Algunas de esas herramientas son: Design Trinking, Sit, Six Sigma, Lean startup, Agile y el desarrollo de nuevos productos.

\section{REFERENCIAS}

Arias, F. G. (2012). El proyecto de investigación. Introducción a la metodología científica, sexta edición. $\underline{U R L}$

Asociación Nacional de Industriales (ANDI). (2019). Colombia: balance 2018 y perspectivas, 2019. $\underline{U R L}$

Bakator, M., Đorđević, D. \& Ćoćkalo, D. (2019). Modelling the Influence of Product Development on Business Performance and Competitiveness in Manufacturing Enterprises. Technical Gazette, 26(6), 1628-1634. https://doi.org/10.17559/TV-20181102185923

Banco Interamericano de Desarrollo (BID). (2016). Competitividad, tecnología, e innovación. URL

Busom, I. (1993). Los proyectos de I+D en las empresas: un análisis empírico de algunas de sus características. Revista Española de Economía, 10(Extra 1), 39-65.

Chasqui Uribe, I. \& Soto Valderrama, M. V. (2016). Impacto de la Innovación en la industria manufacturera América Latina, Colombia y Valle del Cauca. URL

Cegarra, S. (2012). Los métodos de investigación. Díaz de Santos.

Cerda Gutiérrez, H. (1993). Los elementos de la investigación. Búho.

Colombia. Congreso de Colombia. Ley 1838 de 2017. Por la cual se dictan normas de fomento a la ciencia, tecnología e innovación mediante la creación de empresas de base tecnológica (spin offs) y se dictan otras disposiciones. 6 de julio de 2017. URL

DataiFX. (2017). Estas son las 12 empresas más innovadoras en Colombia según la ANDI. URL

Díaz Rodríguez, L. V. (2006). Gestión del conocimiento y tecnologías de la información y comunicaciones. Revista Escuela de Administración de Negocios, (58), 41-60. URL

Durán, X. H., Ibáñez, R., Salazar, M. y Vargas, M. (2000). La innovación tecnológica en Colombia. Características por sector industrial y región geográfica. Colciencias.

Egbu, C. O. y Young, B. A. (1998). Innovation management for refurbishment. En CIB W70 International Symposium on Management, Maintenance and Modernization of Building facilities: the way ahead into the millennium. Singapore.

Fernández, C., Baptista, P. y Hernández, R. (2003). Metodología de la investigación. McGraw Hill. 
Gallardo-Vázquez, D., Valdez-Juárez, L. E., \& Castuera-Díaz, Á. M. (2019). Corporate Social Responsibility as an Antecedent of Innovation, Reputation, Performance, and Competitive Success: A Multiple Mediation Analysis. Sustainability, 11(20), 1-28. https://doi.org/10.3390/su11205614

García González, F. (2012). Conceptos sobre innovación. Contribución al análisis PEST (política, economía, sociedad, tecnología) "plan estratégico 2013-2020". URL

Griffith, R., Redding, S. y Van Reenen, J. (2004). Mapping the two faces of RyD: productivity growth in a panel of OECD industries. Review of Economics and Statistics, 86(4), 883-895. https://doi.org/10.1162/0034653043125194

INNpulsa. (2019). Colombia vive la era del emprendimiento y la innovación. URL

INNpulsa, Colombia. (2018). Colombia avanza dos puestos en el índice Global de Innovación y es reconocida por primera vez como "Innovation Achiever". URL

López Riveros, E. A. (2013). Análisis de productividad de las Pymes en la política de la Competitividad. URL

Maldonado, J. (2018). Metodología de la investigación social. Paradigmas: cuantitativo, cualitativo, socio-crítico, complementario. Ediciones de la U.

Malaver Rodríguez, F. y Vargas Pérez, M. (2004). El comportamiento innovador en la industria colombiana: una exploración de sus recientes cambios. Cuadernos de Administración, 17(27), 33-61. URL

Martínez Villaverde, L. (2010). Gestión del cambio y la innovación en la empresa: un modelo para la innovación empresarial. URL

Morales, M. E., Ortiz Riaga, C. y Arias Cante, M. A. (2012). Factores determinantes de los procesos de innovación, una mirada a la situación en Latinoamérica. Revista Escuela de Administración de Negocios, (72), 148-163. https://doi.org/10.21158/01208160.n72.2012.573

Morales M. y León A. (2014). Adiós a los mitos de la Innovación. URL

Nawas Khan, S., Hussain, R. I., -Ur-Rehman, S., Maqbool, M. Q., Engku ALI, E. I., \& Numan, M. (2019). The mediating role of innovation between corporate governance and organizational performance: Moderating role of innovative culture in Pakistan textile sector. Cogent Business \& Management, 6(1), 1631018. https://doi.org/10.1080/23311975.2019.1631018

Oppenheimer, A. (2014). iCrear o morir! La esperanza de América Latina y las cinco claves de la innovación. Penguin Random House.

Pinto, H., Nogueira, C., \& Domínguez-Gómez, J. A. (2019). Sistemas de innovación y resiliencia regional: un análisis de redes. Revista Española de Sociología, 28(3-Sup1), 35-52. https://doi.org/10.22325/fes/res.2019.27 
Ponti, F. y Ferrás, X. (2008). Pasión por innovar: un modelo novedoso que incentiva la creatividad empresarial. URL

Ponti, F. (2010). Los 7 movimientos de la innovación. Norma.

Revista Dinero. (2018). El ranking de las 5000 empresas más grandes de Colombia. Dinero. URL

Robayo Acuña, P. V. (2016). La innovación como proceso y su gestión en la organización: una aplicación para el sector gráfico colombiano. Suma de Negocios, 7(16), 125-140. https://doi.org/10.1016/j.sumneg.2016.02.007

Sivam, A., Dieguez, T., Pinto Ferreira, L., \& Silva, F. J. G. (2019). Key settings for successful Open Innovation Arena. Journal of Computational Design and Engineering, 6(4), 507-515. https://doi.org/10.1016/i.jcde.2019.03.005

Ursúa Lasheras, A. (2019). La importancia de la innovación en la transformación de la industria. $\underline{U R L}$ 